\title{
Re: Development of an Emergency Medicine Wellness Curriculum
}

To the Editor:

Tn 2018, AEM Education and Training published the I authors' article entitled "Development of an Emergency Medicine Wellness Curriculum," detailing a multifaceted wellness curriculum that included bimonthly structured didactic presentation, corresponding nondidactic elements, individualized interactive instruction (III) assignments, and additional Internet-based resources. ${ }^{1}$ This curriculum was subsequently implemented at five emergency medicine residencies across the country from March 2017 to February 2018. At the conclusion of the curriculum, the authors performed a postintervention survey at the participating sites to determine resident perceptions of the curriculum. Specific survey questions included: "Did you find the wellness curriculum offered by your program this year to be beneficial to your overall feelings of wellness?"; "What were the most useful aspect of the curriculum for you?"; and "Besides resources offered by your residency program, what other resources did you access over this year with the goal of improving your wellness?"

There were several interesting findings to the survey that may aid educators in the development of future wellness interventions. A total of 93 of 174 (53\%) residents completed the survey. A majority of resident respondents (53.8\%) found the wellness curriculum to be moderately or highly beneficial to their overall feelings of wellness. Respondents reported that the most useful aspects of the curriculum were dedicated time for resident bonding (63\%), dedicated time for residency wellness activities (48\%), and a wellness retreat $(41 \%)$. Far fewer residents found individualized interactive instruction (5\%), designating wellness champions (3\%), and assigned readings about wellness (2\%) to be useful. Routinely accessed external resources included setting personal fitness goals (75\%), regular participation in a hobby $(53 \%)$, and yoga $(28 \%)$. Fewer residents participated in volunteer activities (12\%), meditation (10\%), counseling (9\%), and external wellness retreats (9\%).

Our survey study demonstrated that for residents, the most valued components of the wellness curriculum centered on themes of social wellness, including dedicated time for resident bonding, and for residency wellness activities including a retreat. Items that may have been perceived to entail additional work, such as III, assigned readings, and designation of a wellness champion were not as well-received. As educators address the ACGME common program requirement concerning resident well-being, it is important to develop curricula that incorporate elements that are valued by resident physicians.

Kelly Williamson, MD

(kellywilliamsonmd@gmail.com)

University of Illinois at Chicago, Chicago, IL; Department of Emergency Medicine, Advocate Christ Medical Center, Oak Lawn, IL

Patrick M. Lank, MD, MS

Feinberg School of Medicine, Northwestern Emergency Medicine Residency, Northwestern University, Chicago, IL

Elise O. Lovell, MD University of Illinois at Chicago, Chicago, IL; Department of Emergency Medicine, Advocate Christ Medical Center, Oak Lawn, IL

The Emergency Medicine Education Research Alliance (EMERA)

Supervising Editor: John H. Burton, MD

\section{Reference}

1. Williamson K, Lank PM, Lovell EO; Emergency Medicine Education Research Alliance. Development of an emergency medicine wellness curriculum. AEM Educ Train 2018; 2:20-5. 